\title{
Management of malignant pleural effusions
}

Pleural effusions are a common development in patients with cancer, often posing a considerable management problem. From $28 \%$ to $61 \%$ of effusions seen in a general hospital are malignant, ${ }^{1-3}$ and up to half of patients with breast cancer will develop an effusion during the course of their illness. ${ }^{34}$ Other cancers in which pleural effusions occur frequently include lung cancer, ovarian cancer, and the lymphomas. ${ }^{35}$

The prognosis of patients with malignant effusions is generally poor, with a reported 30 day mortality of 29 $50 \%$. ${ }^{6}$ Many patients, however, particularly those with breast cancer, have prolonged survival ${ }^{4}$ and control of the effusion and the related symptoms is an important issue in management.

Several methods have been used to control malignant pleural effusions. They have included repeated needle thoracentesis, external radiotherapy, systemic chemotherapy, tube thoracostomy with or without instillation of cytotoxic drugs or sclerosant agents, and pleurectomy. The great variety of techniques used highlights the fact that no single approach is distinctly superior. Furthermore, published reports deal predominantly with retrospective series, with a large variation in the evaluation criteria used by different authors. This makes comparison of different techniques difficult and underscores the need for randomised studies comparing treatment approaches.

The World Health Organisation has published criteria for assessing tumour response at sites of non-measurable disease. ${ }^{8}$ These criteria define complete response as the "complete disappearance of all known disease for at least four weeks" and partial response as an "estimated decrease in tumour size of $50 \%$ or more for at least four weeks." Although these definitions may be applicable at other sites of non-measurable disease, they are difficult to apply to pleural effusions, particularly in the context of "partial responses." In this setting criteria incorporating the frequency of pleural aspiration may be more meaningful.

The approach to management of malignant effusions varies with the type of cancer and the extent of extrathoracic disease. If systemic treatment has a reasonable probability of producing tumour response in the particular cancer and other symptoms warrant its use, pleural effusions are usually also controlled. ${ }^{9}$ When this is not the case local measures may achieve control of pleural effusions that is occasionally longlasting.

The definitive surgical method of eliminating the pleural space for management of malignant pleural effusion is pleurectomy. Unilateral obliteration of the pleural space does not appear to have any long term consequences-and, interestingly, the lungs of the elephant, lacking a pleural space, do not seem to operate at a disadvantage. Martini $e t$ al performed pleurectomies in a group of 106 patients with cancer, $90 \%$ of whom had recurrent effusions after other methods of treatment. ${ }^{10}$ None had a recurrence of the effusion after pleurectomy and median survival was 16 months. Morbidity $(23 \%)$ and mortality $(10 \%)$ were substantial, however, and this approach cannot be recommended as initial treatment for most patients.

By contrast, the least invasive method of management is thoracentesis. Unfortunately, the benefit of this procedure is usually short lived, with most effusions accumulating again within one month. ${ }^{11}$ This limits its usefulness for the acute relief of symptoms or as a component of instillational sclerotherapy.

Between these two extremes are several methods that have been advocated to control malignant pleural effusions. Older methods, such as external radiotherapy and instillation of radiocolloids or of non-sclerosant cytotoxic agents, have been largely abandoned, because of either lack of efficacy or excessive toxicity, ${ }^{3}$ although mediastinal radiotherapy may be effective in the treatment of effusions in lymphoma patients. ${ }^{11}$

The most commonly used means of treating malignant pleural effusions is drainage followed by instillation of a "sclerosant" agent. The aim of this procedure is to induce a chemical pleuritis, which as it resolves forms a symphysis between the two pleural surfaces, obliterating the pleural space and preventing reaccumulation of the effusion. The method of drainage used varies, some investigators favouring needle thoracentesis and others using tube drainage. ${ }^{3}$ In two (non-randomised) studies ${ }^{411}$ the use of tube drainage resulted in results superior to those of needle aspiration, though similar results have been reported with this latter method. ${ }^{12}$ Irrespective of the method chosen for drainage, it is probably important to drain the pleural space to dryness before instillation of the sclerosing agent, to minimise dilution of the instilled agent and aid in the apposition of the pleural surfaces. ${ }^{13}$

A wide range of "sclerosants" have been used after drainage, including tetracycline, bleomycin, quinacrine, nitrogen mustard, adriamycin, and talc. Recently, mitoxantrone has been reported as being useful in this setting. ${ }^{14}$ There have been few randomised trials comparing any of these agents. Bayly et al reported that tetracycline was as effective as quinacrine and had fewer side effects. ${ }^{12}$ In another randomised study, ${ }^{15}$ comparing adriamycin, tetracycline, and nitrogen mustard, little difference was found between adriamycin and tetracycline for control of pleural effusions, though both were more effective than nitrogen mustard. Tetracycline, however, had the lowest incidence of side effects. Hausheer and Yarbro ${ }^{3}$ imposed response criteria retrospectively on several trials of instillation treatment. They concluded that talc was probably the most effective sclerosant but that this was offset by greater 
morbidity than with other agents, making it unsuitable as first line treatment. Tetracycline $(15 \mathrm{mg} / \mathrm{kg}$ or $1 \mathrm{~g})$ and bleomycin ( $1.25 \mathrm{mg} / \mathrm{kg}$, not to exceed $40 \mathrm{mg}$ in the elderly) were recommended, control of effusions being expected in $70 \%$ and $85 \%$ of cases for one month. Quinacrine, though possibly more effective than these agents, has the major drawbacks of increased toxicity and the necessity of multiple administrations.

Treatment using biological agents has also been reported. ${ }^{16-18}$ Corynebacterium parvum has been reported to be more effective than nitrogen mustard in a randomised trial, ${ }^{16}$ in which it was successful in all treated patients. More recently, $\beta$ interferon has been proposed as a potentially useful agent in the management of malignant effusions. ${ }^{18}$ The initial results, however, are disappointing, with an overall response rate of only $38 \%$, and with two thirds of the patients requiring three treatments. ${ }^{18}$

In the absence of data from randomised trials comparing the available methods, the treatment of malignant pleural effusions should be guided by the experience of the clinicians concerned. Some broad guidelines, however, are useful. Firstly, the treatment goal in patients with malignant effusions is palliative in most cases, and the mere presence of fluid in the pleural cavity is not itself an indication for intervention. The severity of symptoms and the rate of fluid reaccumulation are important factors in determining the appropriate management. Secondly, if systemic treatment is not justifiable initial management ought to comprise drainage to dryness, by either tube or repeated aspiration, perhaps followed by sclerotherapy with either bleomycin or tetracycline. The use of narcotic premedication or the addition of lignocaine to the sclerosant solution may reduce local pain for the patient. Finally, where reaccumulation occurs after attempted sclerosis, and provided that the patient has a life expectancy of some weeks or months, talc pleurodesis or pleurectomy may be attempted.

Future investigation requires the adoption of a standard set of response criteria, such as those used by Hausheer and Yarbro, ${ }^{3}$ so that some comparison may be made between series. In addition, measures of quality of life should be included in the assessment of treatment outcome, as this is a palliative procedure. There is an urgent need for prospective, randomised studies comparing different forms of treatment on the basis of relevant end points, rather than further large reviews of individual clinicians' experience.

MHN TATTERSALL MJ BOYER

Department of Medical Oncology

Royal Prince Alfred Hospital

Camperdown 2050

Sydney, Australia

Reprint requests to Professor Tattersall.

1 Light RW, Macgregor MI, Luchsinger PC, Ball WC. Pleural effusions: the diagnostic separation of transudates and exudates. Ann Intern Med diagnostic separat
1972;77:507-13.

2 Tinney WS, Olsen AM. The significance of fluid in the pleural space: a study of 274 cases. $J$ Thorac Surg 1945;14:248-52.

3 Hausheer FH, Yarbro JW. Diagnosis and treatment of malignant pleural effusion. Semin Oncol 1985;12:54-75.

4 Fracchia AA, Knapper WH, Carey JT, Farrow JH. Intrapleural chemotherapy for effusion from metastatic breast carcinoma. Cancer 1970;26: 626-9.

5 Leff A, Hopewell PC, Costello J. Pleural effusion from malignancy. Ann Intern Med 1978;88:532-7.

6 Chernow B, Sahn SA. Carcinomatous involvement of the pleura: an analysis of 96 patients. Am J Med 1977;63:695-702.

7 Gravelyn TR, Michelson MK, Gross BH, Sitrin RG. Tetracycline pleurodesis for malignant pleural effusions. A 10 year retrospective study. Cancer 1987;59:1973-7.

8 Miller AB, Hoogstraten B, Staquet M, et al. Reporting results of cancer treatment. Cancer 1981;47:207-14.

9 Tattersall MHN. Intracavitary treatment of malignant pleural effusions. Chemioterapia 1982;1:288-92.

10 Martini N, Bains MS, Beattie EJ. Indications for pleurectomy in malignant effusion. Cancer 1975;35:734-8.

11 Anderson CB, Philpott GW, Ferguson TB. The treatment of malignant pleural effusions. Cancer 1974;33:916-22.

12 Bayly TC, Kisner DL, Sybert A, Macdonald JS, Tsou E, Schein PS. Tetracycline and quinacrine in the control of malignant pleural effusions. Tetracycline and quinacrine in the control of

13 Malden LT, Tattersall MHN. Malignant effusions. $Q J$ Med 1986;58: 221-39.

14 Musch E, Seitzer D, Chemaissani A, et al. Intracavitary instillation of mitoxantrone for palliative therapy of malignant effusion [abstract 55]. In: Proceedings of workshop on progress in regional cancer therapy I. Vienna, 1988.

15 Kefford RF, Woods RL, Fox RM, Tattersall MHN. Intracavitary adriamycin, nitrogen mustard and tetracycline in the control of malignant effusions. Med J Aust 1980;ii:447-8.

16 Millar JW, Hunter AM, Horne NW. Intrapleural immunotherapy with Corynebacterium parvum in recurrent malignant pleural effusions. Thorax Corynebacterium

17 Serrou B, Cupisoll D. Épanchements pleuraux. Traitement local par Corynebacterium parvum. Nouv Presse Méd 1980;25:1777-8.

18 Rosso R, Rimoldi R, Salvati F, et al. Intrapleural natural beta interferon in the treatment of malignant pleural effusions. Oncology 1988;45:253-6. 